\title{
Healthy Culture of Aquatic Animals and Development of Green Fishery Medicine
}

\author{
Guangjun Wang \& Xidong Mou \\ Pearl River Fishery Research Institute, Chinese Academic of Fishery Science \\ Guangzhou 510380, China
}

Tel: 86-20-8161-6311 E-mail: gjwang@prfri.ac.cn

Guangping Yin \& Qingshen Liu

South China Agricultural University

Guangzhou 510642, China

This paper was supported by Chinese National Project of Advanced and New Technology (863) (No. 2007AA10Z239), Earmarked fund for Modern Agro-industry Technology Research System(No. nycytx-49), Open Fund of Key Laboratory of Genetic Breeding and Aquaculture Biology of Freshwater Fishes, Ministry of Agriculture)(No. BM2007-03), Science and Technology Plan Project of Guangdong Province (No. 2009B020315008),National key technologies $R$ \&D Program of China (No. 2006BAD03B0102).

\begin{abstract}
Since reforming and opening to the outside world, Chinese aquiculture has developed very quickly. The total output of aquiculture has been ranking first in the world over ten years, but it still has many problems. In this article, many problems are listed about Chinese aquiculture, which has seriously influenced the quality of aquatic products and destroyed the whole aquiculture ecological environment, so it is imperative under the situation to implement healthy culture. At the same time, the actuality of Chinese healthy culture is analyzed, and healthy culture has been developed in freshwater and sea water. Healthy culture comes down to many aspects, and it is a very important part to reasonably use fishery medicines. The using of fishery medicines should start from many aspects including medicine materials, cause of disease, environment, aquatic animals, and human health, and only to use medicines intentionally and effectively can achieve the effect of preventing and treating diseases. The green fishery medicine is one most effective development direction at present to use medicines reasonably. Green fishery medicines include fishery vaccine, Chinese herbal medicine preparation, animalcule preparation, and biologic fishery medicines.
\end{abstract}

Keywords: Aquatic animals, Healthy culture, Green fishery medicine

\section{Necessity to implement healthy culture}

The total output of Chinese aquiculture has been ranking first in the world over ten years. The total output of 2000 had exceeded 40 million tons, and this number had achieved 48.9 million tons in 2008. But most Chinese aquiculture modes still are traditional aquiculture modes. With the further development of the aquiculture, the disadvantages of this mode have been represented increasingly, and it can not accord with the development requirement of aquiculture in China any more. Though the traditional aquiculture mode can increase the total output of aquiculture by increasing the aquiculture area, but the aquiculture benefit has descended obviously and the quality of aquatic products decreases significantly. The discharge of aquiculture nutriment and the use of chemical medicines will pollute water and deteriorate the environment. The diseases of main aquiculture breeds are serious and prevail explosively. In the seawater aquiculture, the artificial damage to the mudflats and aquiculture sea area have induced large-area red tides, serious deterioration of seacoast ecological environment, and decreasing aquatic biologic diversity (Shang, 2001, P.43).

As the existences of above disadvantages, though the total output of Chinese aquiculture has been ranking first in the world over ten years, the quality is not satisfactory, and the exports are often refused because of the over-standard animalcule and forbidden antibiotics (Zhao, 2002, P.66-70). The selective examination result of domestic market showed that the qualified rates of the products including shrimp, scallops frozen, and fish could not achieve $50 \%$, and 
the phenomenon of adding excessive additives artificially still exists universally. People still can memorize that the hepatitis A induced by unclean blood clam prevailed in Shanghai in the late of 1980s. In 2000, European Union passed the decision to allow China export aquatic products to European Union, but there were only 159 eligible enterprises in 5000 enterprises which can manufacture aquatic products. The safety of aquatic products has been the most serious problem facing by the aquiculture at present, and in this way, China only can be the "big country of aquiculture", but not the "strong country of aquiculture".

Therefore, people gradually realize the importance of problem, and begin to explore new aquiculture mode, and study new aquiculture technology and method to reduce the pressure of the aquiculture environment and maintain the sustainable development of the aquiculture industry. So the concept of "healthy culture" is proposed and implemented (Cai, 2001, P.54-55 \& Zhao, 2002, P.63 \& Yao, 2002, P.10-13 \& Wang, 2002, P.5 \& 36 \& Han, 2002, P.25-27 \& Zhu, 2003, P.34-35 \& Dong, 2003, P.24-26).

"Healthy culture" is a new culture concept which was proposed in recent years, and comparing with traditional aquiculture technology and management, it contains more extensive contents, and it not only requires healthy aquiculture culture products to ensure the safety of human foods, but the aquiculture ecological environment should accord with the ecological requirements of aquiculture breeds, and the aquiculture breeds should keep relatively stable breed characteristics. The introduction of this concept is mainly because that the random of Chinese aquiculture technology and management has induced spreading diseases, degenerated breeds, decreasing product quality, and even influenced the healthy safety of foods.

\section{Actuality of Chinese healthy culture}

Fortunately, healthy culture in China has been carrying out (Zhang, 2000, P.19-20 \& Bai, 2002, P.3-5). The Freshwater Fisheries Research Center of Chinese Academy of Fisheries Science has studied the pond dynamics and animalcule biology for a long time, and many aspects such as the beneficial organisms including photosynthetic bacteria and the interior water quality control and disease prevention in the aquiculture system have been developed quite well. The Freshwater Fisheries Research Center of Chinese Academy of Fisheries Science and Nanjing Institute of Geography and Limnology extensively studied the influences of different aquiculture modes to the water environment, and the sustainable development technology and relative mode. Professor Li Sifa of the Key Lab of Chinese Ministry of Agriculture and Shanghai Fisheries University has engaged in the fish seed inheritance improvement, healthy parent strain, and seed selection all along, and has acquired abundant results, for example, the immaterial asset of "Pujiang No. 1" selected by him has achieved 1 billion Yuan. The fishery vaccine study in the Pearl River Fisheries Research Institute of Chinese Academy of Fisheries Science from early indigenous vaccine of grass carp to present subunit vaccine and DNA vaccine all show that the good effect of vaccine in the disease prevention of aquatic animals.

In marine-culture of China, the healthy aquiculture management has been advocated and some corresponding technologies have been developed, especially the disease prevention system has been established, the diagnosis technology has been developed, the using and the development of aquatic medicines are going to standardization, the aquiculture breeding has been emphasized universally, the research of aquiculture capacity and the development of ecological aquiculture all have obtained some initial results (Chen, 2003, P.62-64).

\section{Fishery medicines should be reasonably used in healthy culture}

In the practice, the healthy culture includes five aspects such as seed, culture, water quality, feed, and medicine. And the use of aquatic medicines is a very important part, as the healthy culture requires scientific and reasonable using of medicine in the aquiculture (Wang, 2003, P.1-3). Its intention is to enhance the disease prevention effect of aquiculture animals and the quality of aquatic products. Reasonable use of medicines should start from medicine, disease cause, environment, aquatic animals, and human health to intentionally and effectively use medicines and achieve the effects of preventing and treating diseases (Lin, 2002, P.63-64). Medicines have both positive function and negative function. On the one hand, medicines can prevent and treat diseases or improve the environment and strengthen the constitution of aquatic animals. And on the other hand, if medicines are used more frequently, not only the disease causes will produce drug tolerance to invalidate medicine prevention, but also the aquatic animals will be harmed or stimulated to destroy the microbial environment of aquatic animals.

The reasonable use of medicines should emphasize "giving priority to prevention, and combining prevention and treatment". In the season that diseases prevailing, the medicines which can restrain and kill causes of diseases should be offered periodically according to the prevalence rule of diseases, or the medicines which can enhance the metabolism mechanism of aquatic animals should be used to prevent the occurrence of diseases. The usual method is to mix medicines into feeds, for example, adding some bacteriophages or Chinese herbal medicines (such as isatis root, rhubarb, garlic, and coptis root), and some vitamins and mineral compositions. At present, in the aquiculture, the phenomenon of "emphasizing treatment and ignoring prevention" still exists, and once the diseases come on, medicines are always abused, so the drug tolerance of disease causes will be formed. 
The reasonable use of medicines should also develop and use special aquatic medicines, such as fishery vaccine, animalcule preparation, biologic fishery medicines, and natural Chinese herbal medicines. Most fishery medicines used in aquiculture are composed by human medicines and animal medicines without pertinence, and the residuals of many fishery medicines are very serious, which will seriously threaten the aquatic biological environment and human health in a long time. For the sustainable development of aquiculture and human healthy, it is urgent to study low-poison and strong pertinence fishery medicines without residuals and pollution, especially the development of fishery vaccine and Chinese herbal medicines should be the emphasis of further work. Fishery vaccine and Chinese herbal preparation will not negatively impact the aquatic animals, they are real "green fishery medicines". Study the using and effect fishery vaccine and Chinese herbal medicines is one direction of reasonable use of medicines.

\section{Green fishery medicines}

The so-called "green fishery medicines" means safe and harmless fishery medicines which is the high-technology product combining with agriculture science, environment protection science, nutrition science and health science, i.e. These kinds of medicines which utilize natural medicines and beneficial biology swarms, and adopt modern advanced pharmacy technologies to prevent the diseases of aquatic animals such as fish, shrimp, and shellfish and improve the environment of aquatic animals. It will not destroy the ecological balance of aquatic animals and produce residuals of medicines. It has better prevention effects, and it can not only prevent diseases but also protect the ecological environment. It mainly includes fishery vaccine, natural Chinese herbal medicine preparation, animalcule preparation, and biologic fishery medicines.

\subsection{Fishery vaccine}

The fishery vaccine is the most effective measure to prevent the explosive epidemics of aquiculture. Vaccine can not only prevent bacteria diseases, it is also the unique effective measure to deal with virus diseases. Different with traditional aquatic medicines, vaccine is not kill medicine causes, but strengthen aquatic animals' resistance to some intensive infectious diseases and make them to void these infectious diseases. And most aquatic vaccines need only to be used once in the whole aquatic period.

In 1969, the Pearl River Fisheries Research Institute first developed the aquatic vaccine successfully, which can be extended and used in large area of grass carp (a inactivated vaccine of organization plasm). It can essentially treat harmful explosive epidemic diseases such as broken gill, read skin, enteritis, or bleeding, and enhance the survival rate of grass carp in pond aquiculture to over $85 \%$.

After that, the aquatic scientists of China also developed many aquatic vaccine production technologies including inactivated cell vaccine, weak poison activated vaccine, molecule vaccine, and gene engineer vaccine.

The gene engineer vaccine is the vaccine prepared by abstractubg antigenic determinants on the cytoderm of nosogenetic bacterium with modern biologic technology. It is harmless, and has high immunity protection rate, and strong specificity, and only two gamma needs to be injected for one grouper. And the vaccine can be stored for five years in normal temperature. It is also easily to be produced and transported, and the industrialized production ability has been formed at present.

Comparing with above vaccines, the gene engineering vaccine is more safe and reliable, and it has high purity, low cost and large production scale. Lately, Zhongshan University successfully developed the gene engineering vaccine of soft-shelled turtle hydrosphere monad, and the lab immunity protection rate can achieve $100 \%$, and it has been tested in large area (Li, 2000, P.30-32).

Recently, people also successfully developed DNA vaccine (gene vaccine or nucleic acid vaccine). It means that the eukaryotic expression plasmid DNA with coding antigen gene can be incepted by host cell through directly being inoculated into inoculums, and express corresponding antigens by transcribing and translating, and generate the immunity answer of this antigen by different approaches to stimulate organisms and achieve the effect of immunity. The preparation method of vaccine is simple and fit to produce in large scale, and it has high efficiency and stability with low costs. It can be expressed for a long time in the organism, and continually stimulate the immunity system of the organism, and compose multivalent vaccine which can generate the immunity protection function aiming at multiple antigen expressions (Yin, 2001, P.87-90 \& Bai, 2001, P.57-59).

\subsection{Chinese herbal medicine preparations}

Natural Chinese herbal medicines have many characters such as cheap cost, low poison, little side effect and difficult generation of drug tolerance and some components can not only resist bacterium, but immunize virus, and this kind of medicines can improve the immunity status of the organism, and enhance the anti-bacteria ability. The advantages of Chinese herbal medicines to prevent fishery diseases include abundant medicine sources, cheap costs, extensive function, treating both principal and secondary aspect of disease, safety and low poison, and difficult generation of drug tolerance, and can also obviously enhance the production performance of fish, and increase the economic benefits of 
aquiculture (Sha, 2003, P.60 \& Li, 2003, P.29-31 \& Sun, 2002, P.37-38 \& Wang, 2001, P.17-18).

Natural Chinese herbal medicines have wide application foreground. For example, the gallnut and sanguisorba can prevent and treat Edwardsiella tarda, the coptis root and phellodendron can prevent and treat the disease of pasteurellosis, the gallnut (dousing) and Chinese tallowtree leaf (mixing) and folium eucalypti (dipping) can prevent the bacterial gill-rot disease, and the garlic, wolf's milk, polygonum hydropiper and creat can prevent and treat the bacillary enteritis. In recent years, the scientists of Pearl River Fisheries Research Institute using Chinese herbal composite preparation to treat the white-soleplate disease of turtle and the red leg disease of white-leg shrimp, the result is very well.

In cultre, China is one of the most developed country in the world, and the founder of natural Chinese herbal medicines, and the headstream and village of Chinese herbal medicines. When the natural Chinese herbal medicines extensively begin to rise in the world, China should push the research and application to a new height especially in the aquiculture industry, and use the high technology to change the traditional industry and improve the ecological environment, prove the mechanism of Chinese herbal medicines, and establish the system of R \& D and standards to initiate the fishery disease prevention medicines and feed additives and aquatic science and technology with Chinese characteristics.

\subsection{Microorganism preparations}

To prevent and treat the diseases of aquatic animals by chemical medicines or antibiotics only is temporary measure, and the broad-spectrum antibiotic can kill or restrain sensitive bacterium and keep pathogenic bacterium with drug tolerance, it also can destroy or disturb the ecological balance of the original normal animalcule region of waters, and increase the opportunity that aquatic animals infect pathogens. The residual of antibiotic in the organism will finally harm human, and the ecological prevent and treatment is a good measure to solve problems, so the research and development of animalcule preparation begin to occur. In the ecology, it mainly study the function and characters of animalcule swarm, optimize the ecological structure of aquiculture water area, and develop the aquiculture production in good circulation, and obtain larger economic, ecological and social benefits (Gui, 2001, P.86-87 \& Liu, 2002, P.17-18 \& Yang, 2000, P.23-24 \& Gong, 2003, P.83-84). Following animalcules have been developed at present.

(1) Photosynthetic bacterium. It is the animalcule to reproduce by light as the energy. And its mycelium contains abundant proteins, various vitamins, biotins, carotenoids, coenzyme $\mathrm{Q}$ and other living activated materials. In addition, it has special physiological function, i.e. it can absorb the ammonia-azote, nitrite, sulfureted hydrogen, and organic acid to eliminate the harmful materials in the waters and purify the water, and the pathogenicbacteria will not survival any more.

(2) Nitrobacteria. It belongs to self-nutritional bacterium, and includes two different metabolic swarms, i.e. nitrosomonas and nitrobacter. Both of them are aerobic bacteria which can grow in oxygenic waters, and play important function to purify waters. One important function of nitrobacteria is to oxidate poisonous ammonia to innocuous nitric acid for the growth of aquatic animals.

(3) Mixed bacterium (composite animalcule). Mixed bacterium is not the name of a kind of bacterium, but the name of a kind of animalcule preparation because this preparation is composed by multiple beneficial activated animalcules which can decompose organisms and purify waters, and the method which adopts single animalcule (such as photosynthetic bacterium and nitrobacteria) to control and purify waters has certain limitation, so multiple animalcules bacterial strains existing in natural environment are selected and cultivated to form the mixed bacterium preparation in the world (Li, 1999, P.34-35).

\subsection{Biologic fishery medicines}

The biologic pharmacy is the process that applies the biologic engineering technology into the domain of pharmacy, and the main method is the gene engineering, i.e. utilizing the monoclonal antibody organization cultivation technology to cut, insert, connect, and rebuild DNA for acquiring medical biologic products. The biological product takes animalcule, vermin, animal toxin, and biologic organization as the starting materials, adopts the biologic technique or the separation purification technology to prepare, and use the biology technology and the analysis technology to control the middle production and the product quality to make biologic activities including bacterin, vaccine, toxin, toxoid, blood serum, blood products, immunity preparation, cell gene, antigen, monoclonal antibody and gene engineering product, DNA rebuilt products, and IVD. At present, biological products include gene engineering medicine, biologic vaccine, and biologic diagnosis reagent.

The fishery medicine manufactured in the biologic pharmacy process is biologic fishery medicine. At present, the biologic medicines have been widely applied in many diseases such as cancer, aids, coronary heart disease, multiple sclerosis, anemia, hypogenesis, diabetes, heart failure, haemophilia, cystic fibrosis and some infrequent genetic diseases. And the application of alumen, polypeptide, enzyme, hormone, vaccine, cell growth factor, and monoclonal antibody manufactured by the recombinant DNA technology in the aquiculture have shown good foreground (Yang, 1999, P.44-45). 


\section{References}

Bai, Junjie \& Yexing. (2001). DNA Vaccine and Its Progress of Application Studies in Aquaculture. Journal of Shanghai Fisheries University, No. 10(1), P. 57-59.

Bai, Yisheng. (2002). Present and Develop of Installation Fisheries and Health Culture in China. Freshwater Fisheries, No. 32(1), P. 3-5.

Cai, Xuefeng \& Luo, Lin. (2001). Foreground of Fishery Healthy Aquiculture. Scientific Fish Farming, No. 10, P. 54-55.

Chen, Shijie. (2003). Health Aquaculture in Seawater. Journal of Fujian Fisheries, No. 1, P. 62-64.

Dong, Zhiguo. (2003). The Health Raise of Aquiculture. China Feed, No. 11, P. 24-26.

Gong, Qingsong. (2003). Application of Animalcule Preparation in Aquaculture. China Fisheries, No. 6, P. 83-84.

Gui, Xiong. (2001). Research and Application of Animalcule Preparation for Aquatic Animals. China Fisheries, No. 12, P. 86-87.

Han, Maosen \& Zhang, Xiaoling. (2002). A Study on Problems of Healthy Culture. Modern Fisheries Information, No. $17(3)$, P. 25-27.

Li, Chunzhi \& Ye, Qiaozhen. (2000). Application of Genetic Vaccine in Control of Diseases of Trionyx Sinensis. Freshwater Fisheries, No. 30(5), P. 30-32.

Li, Qiaolin. (2003). Application of Chinese Herb-medicine in Aquaculture. Chongqing Fisheries, No. 1, P. $29-31$.

Li, Zhuojia \& Chen, Kangde. (1999). Application of Beneficial Activating Animalcule in the Healthy Culture of Prawn. China Fisheries, No. 11, P. 34-35.

Lin, Kai. (2002). Strengthening the Medicine Management of Eel Culture and Advocating the Healthy Aquaculture of Eel. China Fisheries, No. 9, P. 63-64.

Liu, Furong. (2002). Animalcule Preparation and Health Aquiculture. Guangxi Tropical Agriculture, No. 4, P. 17-18.

Sha, Keguang. (2003). Developing Fishery Chinese Herbal Medicines and Promoting the Healthy Culture of Fish and Shrimp. Scientific Fish Farming, No. 2, P. 60.

Shang, Yuanfu. (2001). Healthy Culture of Aquatic Products and Protection of Water Environment. Chinese Fisheries Economics, No. 6, P. 43.

Sun, Kenian. (2002). Application of Chinese Plant Medicine in Aquatic Farming. Feed Review, No. 2, P. $37-38$.

Wang, Guangjun. (2001). Application of Natural Chinese Herb-medicine in Aquatic Farming. Fuying Feed, No. 9, P. 17-18.

Wang, Yongqiang. (2003). Health Aquaculture and Safe Medicine. Shandong Fisheries, No. 20(1), P. 1-3.

Wang, Zhihui. (2002). Significance of Healthy Marine Culture. Heibei Fisheries, No. 2, P. 5 \& 36.

Yang, Xianle. (1999). The Objective of Prevention and Cure of Aquaculture Diseases on 21st Century. Freshwater Fisheries, No. 29(2), P. 44-45.

Yang, Xianle. (2000). Micro-ecosystem and the Healthy Culture of Aquatic Animals. Inland Fisheries, No. 25(2), P. 23-24.

Yao, Guocheng. (2002). Healthy Culture Technique for Offshore Fish. Fishery Modernization, No. 3, P. 10-13.

Yin, Guanghui \& Lin, Tianlong. (2001). Progress of Studies on DNA Vaccine for Fish. Journal of Fishery Sciences of China, No. 8(4), P. 87-90.

Zhang, Fanrong \& Hu, Chuanlin. (2000). Achievements of Aquaculture in China and the Ecological Principles for Healthy Culture of Fishes. Reservoir Fisheries, No. 20(6), P. 19-20.

Zhao, Fazhen \& Li, Jian. (2002). Study on Development Strategy of Fisheries Healthy Cultivation and Food Safety. Marine Fisheries Research, No. 23(4), P. 66-70.

Zhao, Yongfeng. (2002). Technology of Healthy Culture. Scientific Fish Farming, No. 8, P. 63.

Zhu, Wenjin \& Mu, Lei. (2003). Methods and Policies of Healthy Culture of Aquatic Products. Henan Fisheries, No. 2, P. 34-35. 